\title{
Review Article \\ Evidence for Maternal-Fetal Genotype Incompatibility as a Risk Factor for Schizophrenia
}

\author{
Christina G. S. Palmer \\ Departments of Psychiatry, and Biobehavioral Sciences and Human Genetics, UCLA Semel Institute, 760 Westwood Plaza, \\ Room 47-422, Los Angeles, CA 90095, USA \\ Correspondence should be addressed to Christina G. S. Palmer, cpalmer@mednet.ucla.edu
}

Received 17 September 2009; Revised 9 February 2010; Accepted 20 February 2010

Academic Editor: Robert Elston

Copyright ( $\odot 2010$ Christina G. S. Palmer. This is an open access article distributed under the Creative Commons Attribution License, which permits unrestricted use, distribution, and reproduction in any medium, provided the original work is properly cited.

\begin{abstract}
Prenatal/obstetric complications are implicated in schizophrenia susceptibility. Some complications may arise from maternalfetal genotype incompatibility, a term used to describe maternal-fetal genotype combinations that produce an adverse prenatal environment. A review of maternal-fetal genotype incompatibility studies suggests that schizophrenia susceptibility is increased by maternal-fetal genotype combinations at the RHD and HLA-B loci. Maternal-fetal genotype combinations at these loci are hypothesized to have an effect on the maternal immune system during pregnancy which can affect fetal neurodevelopment and increase schizophrenia susceptibility. This article reviews maternal-fetal genotype incompatibility studies and schizophrenia and discusses the hypothesized biological role of these "incompatibility genes". It concludes that research is needed to further elucidate the role of RHD and HLA-B maternal-fetal genotype incompatibility in schizophrenia and to identify other genes that produce an adverse prenatal environment through a maternal-fetal genotype incompatibility mechanism. Efforts to develop more sophisticated study designs and data analysis techniques for modeling maternal-fetal genotype incompatibility effects are warranted.
\end{abstract}

\section{Introduction}

Schizophrenia has long been regarded a significant public health issue. This condition, which is estimated to affect more than 2 million persons in the U.S. alone [1], has increased mortality and morbidity compared to the general population $[2,3]$. Individuals with schizophrenia typically suffer from a combination of debilitating symptoms including hallucinations and delusions and treatment-resistant symptoms, such as social withdrawal [4]. The disease affects males and females, although there is evidence to support a number of sex differences in the characteristics of schizophrenia. Compared to females, males may be more likely to develop schizophrenia with $\sim 1.4: 1$ ratio $[5,6]$, have an earlier age at onset [6-8], poorer premorbid social and intellectual functioning, poorer course and medication response, greater structural brain abnormalities [9], more negative, symptoms and fewer affective symptoms [10].

Twin, family, and adoption studies together suggest that schizophrenia is a complex disorder involving both genes and environment $[11,12]$. Further, the evidence suggesting that schizophrenia arises from a process involving prenatal environmental conditions is compelling [13-21]. Numerous case-control studies have demonstrated that individuals with schizophrenia are more likely to have been exposed to prenatal/obstetric complications than their unaffected siblings, normal controls, or psychiatric controls [22], and a meta-analysis of twelve twin studies [11] demonstrated that a nontrivial proportion of liability to schizophrenia can be accounted for by a common or shared environmental effect (11\%; 95\% CI 3\%-19\%). Because the environments of twins are most similar in utero, the role of common environment effects on liability for schizophrenia would most likely occur very early in life. Suspected fetal environmental risks include exposure to maternal stress [23], influenza [24-26], infection [27], famine or prenatal nutritional deficiency $[16,28-31]$, and obstetric complications $[14,15,18,19,32]$. Several reviews $[8,21,33]$, including a meta analysis [19], support the involvement of (i) pregnancy complications such as Rhesus D incompatibility and pre-eclampsia, 
(ii) abnormal fetal growth and development, and (iii) delivery complications that produce fetal hypoxia as risk factors and suggest that obstetric complications contribute approximately a 2 -fold increased risk for schizophrenia. Prenatal/obstetric complications are believed to disrupt normal fetal neurodevelopment and their involvement in schizophrenia susceptibility is consistent with the neurodevelopmental hypothesis of schizophrenia. This hypothesis posits that brain development is disrupted early in life and that subsequent maturational events in combination with other environmental factors leads to the emergence of psychosis during adulthood [34-36].

Support for the important role of prenatal/obstetric complications in schizophrenia also comes from neuroimaging studies. As an example, there is evidence that fetal hypoxia has a differential effect on the hippocampus of schizophrenics and their first degree relatives, suggesting that this temporal lobe region may be sensitive to prenatal environmental conditions [37-39]. Furthermore, anatomical deficits in the medial temporal lobe structures are more severe among patients with schizophrenia who have a history of hypoxia-associated obstetric complications [40]. Hence, not only do these studies suggest that factors that produce prenatal/obstetric hypoxia have an effect on the medial temporal lobe structure, but that genetic liability to schizophrenia also plays a role in predisposing an individual to schizophrenia. This evidence has produced a variety of hypotheses regarding how genetic and environmental influences aggregate to increase susceptibility to schizophrenia, with gene-environment interaction, gene-environment covariation, or direct environmental effects, that is, phenocopy model, as (potentially overlapping) models [41]. To date, there is very little evidence to support a phenocopy model or a gene-environment covariation model to explain the role of prenatal/obstetric complications in schizophrenia, although additional investigation of these models is warranted [41]. In contrast, evidence that prenatal/obstetric complications increase risk for schizophrenia through a gene-environment interaction model is accumulating. In addition to the studies cited earlier [37-40], a recent study found that risk of schizophrenia was greatest among individuals at highest familial liability who were exposed to maternal infection (consistent with an interaction model) [27]. As another example, significant interaction between suspected hypoxia-regulated/vascular-expression genes and serious obstetric complications (predominantly hypoxia) was found to influence risk for schizophrenia [42].

Although the causes for prenatal complications are quite heterogeneous, their diversity does not exclude a final common pathway and there is increasing discussion that the common pathway involves both the immune and vascular systems in the pathogenesis of schizophrenia [43, 44]. In an excellent review of this theory of schizophrenia, Hanson and Gottesman [43] describe a process in which ubiquitous environmental factors that normally trigger genetically-influenced inflammatory response (infection, trauma, hypoxia) in individuals will trigger abnormal inflammatory processes in individuals with particular genotypes at these inflammatory response loci which results in damage to the microvascular system in the brain. This vascular-inflammatory theory not only accommodates the diversity of prenatal complications associated with schizophrenia, but also specifies an interaction between genes and environment. The latter point helps to explain why most people who experience prenatal/obstetric complications do not eventually develop schizophrenia $[19,21]$, and has received empirical support through the increasing number of studies demonstrating gene-environment interactions in schizophrenia [27, 37-40,42], particularly the recent study that identified an interaction between serious obstetric complications and hypoxia-regulated/vascular-expression genes [42].

Not surprisingly, there remains considerable interest in identifying fetal environmental risk factors and elucidating their role in schizophrenia. However, in addition to their heterogeneity, prenatal/obstetric complications can be difficult to document reliably through medical records or maternal recall, making it difficult to test the role of environmental insults in the pathogenesis of schizophrenia. Of interest, there is some evidence that prenatal complications that increase susceptibility to schizophrenia cluster within schizophrenia families [45], which raises the possibility that some of these complications may have a genetic basis and that these risk genes and hence, adverse prenatal environment, can be measured directly through genetic analyses rather than through medical records or maternal recall. A benefit of direct measurement of the adverse prenatal environment through genetic analysis is that it can facilitate hypothesis testing regarding the role of prenatal/obstetric complications in the pathogenesis of schizophrenia.

Maternal-fetal genotype incompatibility, first described by Palmer et al. [47] to describe a mechanism that confers risk for schizophrenia through maternal-fetal genotype combinations which produce a maternal immunological reaction that creates an adverse prenatal environment, is an example of a prenatal/obstetric complication with a genetic basis. As will be described, maternal-fetal genotype incompatibility can occur when maternal and fetal genotypes differ from one another, or when maternal and fetal genotypes are too similar to each other. "Incompatibility genes" for each of these scenarios have been implicated as risk factors for schizophrenia and are reviewed below. Importantly, maternal-fetal genotype incompatibility is explicitly genetic in nature and so has the potential to be measured directly through genetic analyses even years after the adverse prenatal event has occurred.

\section{RHD Maternal-Fetal Genotype Incompatibility as a Risk Factor for Schizophrenia: When Maternal and Fetal Genotypes Differ}

The teratogenic antibody hypothesis [46] posits that a pregnant female can develop antibodies in response to some antibody producing stimulus (e.g., contact with paternal antigens) that can interfere with normal fetal neurodevelopment. One general mechanism that is consistent with 
the teratogenic antibody hypothesis involves maternal-fetal genotype combinations that adversely affect the developing fetus by inducing a maternal immunological attack. This mechanism is a form of maternal-fetal genotype incompatibility [47], where the development of maternal antibodies can be the result of a mother's genotype that is different from the fetus' genotype.

In some cases, a maternal immunological reaction can lead to hypoxic ischemia, a condition found to be associated with schizophrenia $[17,18]$, and hypothesized to trigger abnormal inflammatory processes in individuals with vulnerable genotypes at inflammatory response loci resulting in damage to the micro-vascular system in the brain and increasing risk for schizophrenia [43]. Conditions that can produce fetal or neonatal hypoxia include maternal-fetal genotype incompatibilities at genes that produce red blood cell antigens, such as the RHD locus.

The RHD gene produces a red blood cell antigen called the Rhesus D factor. An individual who is determined to be Rhesus D positive has red blood cells (RBCs) with this antigen, while someone classified as Rhesus D negative lacks this antigen [48]. Individuals who are Rhesus D positive are either homozygous or heterozygous for an allele that produces the antigen (referred to here as $\mathrm{D} / \mathrm{D}$ or $\mathrm{D} / \mathrm{d}$ ). Individuals who are Rhesus D negative are homozygous for a null allele $(\mathrm{d} / \mathrm{d})$. In Caucasian populations, approximately $85 \%$ of individuals are Rhesus D positive [48].

RHD maternal-fetal genotype incompatibility during pregnancy occurs when a pregnant woman is Rhesus $\mathrm{D}$ negative $(d / d)$ and her fetus is Rhesus D positive $(D / d)$. Because the RBCs of a Rhesus D negative pregnant female do not possess the Rhesus D antigen, maternal anti-D (IgG) antibodies are created in response to detection of fetal RBCs in the maternal blood stream [48]. These antibodies destroy the fetal RBCs in the maternal blood stream, cross the placenta, and destroy fetal RBCs. Because RBCs carry oxygen throughout the fetus' body, including the brain, an attack on the fetal RBCs increases risk for fetal hypoxia which could affect developing tissue, including brain tissue. A byproduct of the destruction of RBCs is bilirubin; thus hyperbilirubinemia, or jaundice can occur, as well as kernicterus, which is deposition of bilirubin in the brain [49]. Bilirubin is a known neurotoxin $[50,51]$ to which undifferentiated glial cells are sensitive $[52,53]$, and glial cell abnormalities also have been associated with schizophrenia $[54,55]$.

An infant is said to have Rhesus hemolytic disease of the newborn when clinical complications arise due to the RHD maternal-fetal genotype incompatibility. Because maternal sensitization usually does not occur until delivery of the first RHD incompatible pregnancy, it is not until second- and later-incompatible pregnancies that risk for a maternal immune attack becomes appreciable [48]. Around 1970 prophylaxis against maternal isoimmunization became available [56], which has made a dramatic impact on the morbidity and mortality associated with RHD maternalfetal genotype incompatibility. However, even in an era of prophylaxis, there continue to be cases of Rhesus hemolytic disease of the newborn, either due to lack of prophylaxis use
$[56,57]$, or because its use is not $100 \%$ effective at preventing maternal sensitization $[58,59]$.

Evidence to support involvement of RHD maternal-fetal genotype incompatibility in schizophrenia comes from both nongenetic and genetic studies performed on samples in which individuals with schizophrenia predominantly were born prior to 1970 [15, 19, 47, 60-66] and reviewed in [67]. The nongenetic studies are based on serotype data (Rhesus $\mathrm{D}$ negative, Rhesus $\mathrm{D}$ positive) or evidence of hemolytic disease of the newborn in mother-child pairs $[19,32,60$, $61,63,64,66]$, while the genetic studies are based on genotype data $(\mathrm{D} / \mathrm{D}, \mathrm{D} / \mathrm{d}, \mathrm{d} / \mathrm{d})$ and nuclear families [47, $65,67]$. Collectively, these studies have provided evidence that $R H D$ maternal-fetal genotype incompatibility is a risk factor for schizophrenia with relative risk ranging from 1.4 [32] to 2.26 [47], a magnitude, that is, comparable to the relative risk of schizophrenia due to obstetric complications in general [19] or due to most genes for which an association with schizophrenia has been observed (see $[68,69]$ for reviews). It is remarkable that studies of RHD maternal-fetal genotype incompatibility and schizophrenia that differ in design and population cohort should arrive at similar relative risk estimates; this consistency suggests that the relative risk of schizophrenia due to $R H D$ maternal-fetal genotype incompatibility, although small, is substantively meaningful and worthy of more investigation.

Another way to look at the magnitude of the RHD maternal-fetal genotype incompatibility effect is to compute the population attributable fraction, that is, the number of cases which would not occur if the risk factor is eliminated. Based on formulas found in [70], for RHD maternalfetal genotype incompatibility, the population attributable fraction is $\sim 3 \%$, as estimated from the fraction of cases that have an $R H D$ maternal-fetal genotype incompatibility $(7.8 \%[65])$ and the relative risk due to the incompatibility (using the most conservative estimate of 1.5 in [65]). Based on a population prevalence for schizophrenia of $1 \%$ and assuming that the allele frequencies for the RHD locus are homogeneous in the U.S., this attributable fraction suggests that more than 100,000 schizophrenia cases in the U.S. would not have occurred but for the RHD maternal-fetal genotype incompatibility. This is not a trivial number, and for comparison, it has been estimated that more than 100,000 cases of schizophrenia in the U.S. would not have occurred but for the val allele of the COMT gene [71]. Thus, these two loci, that is, COMT and RHD, could potentially account for an effect of similar size at the population level. Of course, the allele frequencies at the RHD locus differ across populations, with the $\mathrm{d}$ allele being less common in some populations than others (e.g., $P(D)=.76$ in a study conducted in Nairobi [72] compared to $P(D)=.66$ in the Finnish population [73]). Thus, the frequency of Rhesus negative mothers having Rhesus positive children will vary across populations. This variability in allele frequencies will not affect the relative risk of disease due to RHD maternalfetal genotype incompatibility, but will affect the fraction of schizophrenia cases that are attributed to the RHD locus across populations. 
Since most individuals who are exposed to $R H D$ maternal-fetal genotype incompatibility do not develop schizophrenia, it is highly unlikely that exposure to this adverse prenatal environment alone, that is, a phenocopy model, explains risk for schizophrenia. Furthermore, the lack of evidence for violation of Hardy-Weinberg equilibrium in the founder alleles from the family-based $R H D$ genetic studies $[47,67]$ is inconsistent with a gene-"environment" covariation model because it suggests that mate selection in the schizophrenia families occurred independently of RHD genotype, at least among the founders. To date there have been no empirical studies to determine whether the association between RHD maternal-fetal genotype incompatibility and schizophrenia is explained through a gene"environment" interaction model.

There also is emerging evidence in studies based on serotype data and those based on genotype data that risk of schizophrenia due to $R H D$ maternal-fetal genotype incompatibility may depend on offspring sex [61, 66, 67], with a relative risk of 1.64 in male incompatible offspring and 1.07 in female incompatible offspring based on a recent meta-analysis [67]. Furthermore, a nonsignificant trend suggesting that male offspring are at higher risk than female offspring for schizophrenia due to maternal-fetal genotype incompatibility at another RBC antigen locus, $A B O$, has also been identified based on serotype data [66]. $A B O$ maternal-fetal genotype incompatibility occurs when a pregnant woman has type $\mathrm{O}$ blood and her fetus has type $\mathrm{A}$ or B [48]. As with Rhesus D incompatibility, maternal IgG antibodies can be produced against the fetal antigens and result in hemolytic disease of the newborn [74], although in this case the risk is the same for all pregnancies $[75,76]$.

These sex-dependent findings allow for hypotheses that address why the schizophrenia effect of an RBC antigenassociated maternal immune response is so much greater for male offspring compared to female offspring. It is unlikely to be the case that $R H D$ maternal-fetal genotype incompatibility is more likely to occur in pregnancies with male offspring, nor is there evidence that its related condition of hemolytic disease of the newborn is more likely to occur in pregnancies with male fetuses compared to female fetuses. However, there is evidence that the clinical manifestations of $R H D$ maternal-fetal genotype incompatibility are more severe in pregnancies with male fetuses than with female fetuses [77]. Thus one hypothesis is that specific schizophrenia-effects of RHD maternal-fetal genotype incompatibility (hypoxia, hyperbilirubinemia) can affect female fetuses but that they are less likely to surpass the threshold of severity compared to male fetuses (threshold effect).

There also is evidence that the clinical effects of $R H D$ maternal-fetal genotype incompatibility may occur earlier in gestation for male fetuses compared to female fetuses [77]. Coupled with research supporting sex-differences in brain maturational rates [78], with males exhibiting a slower pace of cerebral development compared to females [7], another hypothesis is that male and females are equally vulnerable to the specific effects of RHD maternal-fetal genotype incompatibility, but that these effects must occur at sex-dependent times during development. This hypothesis further suggests that female fetuses may be at increased risk for schizophrenia when subject to prenatal/obstetric complications that produce hypoxia or hyperbilirubinemia, but that these effects must occur earlier in the gestational period to increase their risk of schizophrenia (a timing effect). A third hypothesis is that male fetuses, but not female fetuses, experience schizophrenia effects due to hypoxia or hyperbilirubinemia (a specific effect). Although there have not yet been studies addressing whether risk of schizophrenia due to RHD maternal-fetal genotype incompatibility in male and female fetuses is a function of a threshold effect or a timing effect, there have been studies addressing potential sex differences in rates of hypoxia-related in males and females, with conflicting results $[79,80]$.

The involvement of the RHD gene in the form of maternal-fetal genotype incompatibility as a risk factor for schizophrenia susceptibility is further substantiated by analyses that showed no evidence to support the idea that this locus is simply linked/associated with a nearby schizophrenia susceptibility locus or that this gene acts through the maternal genotype alone [47]. Furthermore, there is empirical evidence consistent with the hypothesized biological mechanism that previous RHD maternalfetal genotype incompatible pregnancies increase risk for maternal isoimmunization in subsequent pregnancies in the two schizophrenia-RHD maternal-fetal genotype incompatibility studies that tested this hypothesis $[61,65]$. Using serotype information, Hollister et al. [61] divided their birth cohort sample into firstborn Rhesus D-incompatible and Rhesus D-compatible males, and second- or later-born Rhesus D-incompatible and Rhesus D-compatible males. Consistent with a birth order effect, they found the rate of schizophrenia among the second- or later-born Rhesus Dincompatible males was significantly higher than the secondor later-born Rhesus D-compatible males (2.6\% versus $0.8 \%$, $P=.05)$; but that there was no significant difference in the rate of schizophrenia between the firstborn Rhesus Dincompatible and $\mathrm{D}$-compatible males $(P=.64)$.

In the second study, Kraft et al. [65] tested hypotheses about a birth order effect using nuclear families with at least one individual with schizophrenia and $R H D$ genotype data. The model in which there is increased risk only to second- or later-born incompatible children fit the data well, with a significant point estimate of 1.7 relative risk to second- or later-born incompatible children $(P=.014)$. The other model that fit the data well assumed an increased risk for all incompatible children regardless of birth order; the point estimate for the relative risk of schizophrenia in this model was 1.5 (lower than the former model). However, one issue with the latter model is that it forced the risk to first-born incompatible children to be identical to risk to later-born incompatible children, and essentially produced an average relative risk across birth order groups. Since the relative risk was estimated at 1.7 in a model that assumed no risk to first-born, and then the relative risk was lowered to 1.5 in a model that averaged over all incompatible children, the authors concluded that the effect of including the first-born incompatible children was to artificially lower the relative risk relative risk estimates of 
RHD maternal-fetal genotype incompatibility for the laterborn children. It is important to note that neither study had information on pregnancies that did not go to full term, for example, spontaneous abortions. The potential effect of this lack of information is to misclassify some RHD maternalfetal genotype incompatible individuals as first-born (and at very low risk from maternal sensitization) when in fact they were later-born and at heightened risk due to previous maternal sensitization. Such misclassification would serve to underestimate the difference between groups and bias results toward the null hypothesis of no birth order effect. In light of the challenges of truly examining a birth order effect with $R H D$ maternal-fetal genotype incompatibility, it is striking that the two schizophrenia studies that chose to test birth order hypotheses found evidence in support of such an effect. However, further examination of this hypothesis is warranted due to the findings that suggest that the risk of schizophrenia associated with RHD maternal-fetal genotype incompatibility is limited to male offspring. In this case, one would expect to observe an increased risk among secondor later-born incompatible males, but not females. This hypothesis has not yet been tested.

The involvement of Rhesus D incompatibility in schizophrenia was initially provided by studies that inferred genotype status through serotype data. Importantly, the evidence from these non-genetic studies provided the impetus for conceptualizing maternal-fetal genotype incompatibility as a more general non-Mendelian mechanism involved in the etiology of complex disorders such as schizophrenia. The first candidate gene study to test the hypothesis of RHD maternal-fetal genotype incompatibility as a risk factor for schizophrenia [47] provided the proof of principle that this non-Mendelian mechanism can be tested with genotype data. Further, it facilitated the development of statistical methods and study designs based on a candidate gene approach and nuclear families for addressing hypotheses about the role of maternal-fetal genotype incompatibility in disease [65, 81-85]. Such innovations are important because, as illustrated in the next section, not all "incompatibility genes" can be inferred through serotype data.

\section{HLA-B Maternal-Fetal Genotype Incompatibility as a Risk Factor for Schizophrenia: When Maternal and Fetal Genotypes Do Not Differ}

Human leukocyte antigens (HLAs) play an important role in the control of immune responses [86] and there has long been a belief that HLAs play a role in schizophrenia susceptibility, although with conflicting results from genetic studies examining the hypothesis of a high risk allele acting through the affected individual's genotype [87]. Another way to conceptualize the role of HLA in schizophrenia susceptibility is to consider its role(s) in pregnancy. There is strong evidence for maternal recognition of paternallyderived fetal HLAs during pregnancy because maternal antibodies against these fetal antigens have been detected [88]. However, maternal recognition of paternally-derived fetal HLAs that differ from maternal HLAs is believed to be beneficial to implantation and maintenance of pregnancy because maternal antibodies to fetal antigens have been observed in a large number of healthy pregnancies. In contrast, lack of maternal recognition, which is the result of paternally derived HLAs that are not perceived as different from the maternal HLAs, may lead to adverse reproductive outcomes [88].

The underlying biological mechanism for poor reproductive outcomes is not yet known, however, an immunological intolerance hypothesis posits that HLA similarity between mother and fetus fails to stimulate an adequate maternal immune response that is necessary for proper implantation and maintenance of pregnancy [89]. There is some empirical evidence that situations where maternal sensitization would not occur, that is, HLA matching between couples or between mother and fetus, increases the risk of fetal loss [89-92], preeclampsia [93-96], low birth weight [97-100], newborn encephalopathy, and seizures [101]. Importantly, low birth weight and preeclampsia are complications that have been associated with schizophrenia $[15,19,102-104]$. The mechanism(s) by which low birth weight or preeclampsia increase risk for schizophrenia is not yet known. However, a current theory regarding preeclampsia hypothesizes that this condition gives rise to abnormal fetal blood flow that results in chronic fetal hypoxia or malnutrition [105] and both of these conditions are associated with schizophrenia $[15,16,28-31,102,103]$. Furthermore, preeclampsia involves a generalized inflammatory response in the mother as a result of the oxidatively stressed or hypoxic placenta [106], and inflammatory processes are hypothesized to damage the microvasular system of the brain $[43,44]$ and increase risk of schizophrenia [43].

Two additional lines of evidence implicate maternal-fetal HLA matching in schizophrenia. First, evidence supporting the relevance of HLA matching to neurodevelopmental disorders comes from a study that found that parents of children with autism were significantly more likely to share at least one HLA-A, $-B$, or $-C$ antigen in common compared with parents of unaffected children [107]. Second, circumstantial evidence supporting the relevance of HLA matching specifically to schizophrenia comes from the literature on mate selection and the literature on olfaction in schizophrenia. Specifically, in the mate selection literature there is some evidence to support disassortative mate selection with respect to HLA loci [108-111] and that olfaction plays a role in this process $[109,110,112]$. However, studies of individuals with schizophrenia and their unaffected first degree relatives reveal impairments in olfaction [113-116]. Hence, mate selection in this subgroup of individuals may be less likely to be guided by the ability to "sniff out" a mate with HLA dissimilarity, and thus more likely to result in the construction of couples with HLA similarity for whom maternal-fetal HLA matching is more likely to occur.

Because risk of schizophrenia is associated with prenatal/obstetric complications, including preeclampsia and low birth weight, maternal-fetal HLA matching has been associated with these and other pregnancy/obstetric complications, maternal-fetal HLA matching has been observed 
in another neurodevelopmental disorder, and maternalfetal HLA matching may occur more frequently in families of individuals with schizophrenia for biological reasons, a candidate gene study was conducted to assess maternal-fetal genotype incompatibility, that is, matching, at the HLA$A,-B$, and $-D R B 1$ loci as a risk factor of schizophrenia [117]. For this study, Palmer and colleagues hypothesized that maternal-fetal genotype incompatibility increased risk of schizophrenia through a general allele-matching phenomenon rather than through specific allele combinations. For each locus, mother and offspring were considered to match if the offspring's alleles were identical to the maternal alleles or if the offspring's alleles were a subset of the maternal alleles. In either of these cases, maternal sensitization to fetal antigens would not occur because they would be perceived to be the same as the maternal antigens. The maternal-fetal genotype incompatibility test for multiple siblings [65] was modified to accommodate analyses involving a general allelematching phenomenon and missing parental genotypes [82]. There was no evidence for violation of Hardy-Weinberg equilibrium in the founder alleles, consistent with random mating with respect to these three loci. There was no evidence for $H L A-A$ or $-D R B 1$ maternal-fetal genotype matching effect on schizophrenia. In contrast, there was significant evidence for an HLA-B maternal-fetal genotype incompatibility effect $(P=.01)$ where inspection of the parameter estimates revealed that maternal-fetal genotype matching produced a higher risk for female offspring (1.74, 95\% CI: 1.22-2.49) than for male offspring (1.11, 95\% CI: 0.76-1.61). Of note, in the mate selection literature, HLA-B appears to be particularly influential [118].

As this is the first study to demonstrate an association between $H L A-B$ matching and schizophrenia, much more research is needed to determine the mechanism through which this form of maternal-fetal genotype incompatibility increases risk for schizophrenia. One possibility is that HLA$B$ matching increases risk for adverse reproductive outcomes such as preeclampsia or low birth weight. This hypothesis could be tested by examining prenatal and birth records in a sample of females with schizophrenia stratified by $H L A-B$ matching status, and comparing the rates of preeclampsia, low birth weight, and other pregnancy/obstetric complications between the two groups. It also currently is unclear why female offspring would be more vulnerable to effects of HLA-B matching than male offspring. One possibility is that female fetuses are more likely to survive the putative effects of $H L A-B$ matching, such as preeclampsia [119] and hence to be observed in a study, than male offspring. Although the sex-dependent finding is intriguing in light of the work demonstrating that RHD maternal-fetal genotype incompatibility as a schizophrenia risk factor is limited to males [67], replication and investigation of hypothesized clinical manifestations of HLA-B matching (low birth weight, preeclampsia, other complications) are warranted because other published studies reveal conflicting results regarding sex differences in the rates of low birth weight and preeclampsia among individuals with schizophrenia [102, 104]. Because the firstborn child of a couple is at highest risk for preeclampsia, one could also seek further evidence in support of an HLA-B matching-preeclampsia relationship by testing for a birth order effect.

Future research must provide additional evidence for an association between $H L A-B$ matching and schizophrenia, determine if there are clinical outcomes of $H L A-B$ matching, for example, prenatal/obstetric complications, whether HLA$B$ matching increases risk through a phenocopy model or a gene-"environment" interaction model, or is simply associated through a gene-"environment" covariation model, and determine the basis for a sex-dependent risk. It will be particularly important to distinguish between a gene"environment" covariation model and a gene-"environment" interaction model given the a priori basis for expecting higher rates of $H L A-B$ matching in schizophrenia as a function of olfaction deficits.

\section{Future Research}

Based on this review, there are a variety of hypotheses that could be tested in future research to further elucidate the role of RHD and HLA-B maternal-fetal genotype incompatibility in schizophrenia. One important area of research would focus on conducting studies that add to the evidence that these maternal-fetal genotype incompatibilities are risk factors for schizophrenia. As examples, since RHD maternal-fetal genotype incompatibility is genetic in origin, one would expect more clustering of schizophrenia in families with $R H D$ maternal-fetal genotype incompatibility than in schizophrenia families without RHD maternal-fetal genotype incompatibility. The same hypothesis holds for $H L A-B$ maternal-fetal genotype incompatibility. If $R H D$ maternal-fetal genotype incompatibility is a risk factor specifically for males, one would expect to observe that schizophrenia risk is associated with a birth order effect with male offspring exposed to $R H D$ maternal-fetal genotype incompatibility, but not for female offspring. If $H L A-B$ matching is involved in predisposition to pre-eclampsia, low birth weight, or other prenatal/obstetric complication, one would expect higher rates of these prenatal/obstetric complications in individuals with schizophrenia with HLA$B$ maternal-fetal genotype matching compared to those without $H L A-B$ maternal-fetal genotype matching. If $H L A-B$ matching is involved specifically in predisposition to preeclampsia, one would expect to observe that schizophrenia risk is associated with a birth order effect with female offspring exposed to $H L A-B$ maternal-fetal genotype incompatibility, but not for male offspring.

A second area of research would focus on if/how the maternal-fetal genotype incompatibility integrates with genetic liability for schizophrenia (phenocopy, gene-environment covariation, and gene-environment interaction). The phenocopy and gene-environment covariation models are unlikely to explain the association between RHD maternal-fetal genotype incompatibility and schizophrenia. However, one possible explanation for the finding that most people with a history of Rhesus D incompatibility do not develop schizophrenia is that the schizophrenia-producing effect of RHD maternal-fetal genotype incompatibility manifests only in individuals with genetic predisposition to schizophrenia. 
This is a gene- "environment" interaction hypothesis. If this is the case, then one would expect different risks for schizophrenia based on family history and RHD maternal-fetal genotype incompatibility, with greatest risk of schizophrenia among genetically high risk individuals who are exposed to RHD maternal-fetal genotype incompatibility. Following the recent work of Clarke et al. [27], one could test for synergism between RHD maternal-fetal genotype incompatibility and family history of psychosis by comparing the rates of schizophrenia across four groups: no RHD maternal-fetal genotype incompatibility and no family history of psychosis, $R H D$ maternal-fetal genotype only, family history of psychosis only, and RHD maternalfetal genotype incompatibility and positive family history of psychosis. The same interaction hypothesis could be tested for HLA-B maternal-fetal genotype incompatibility; however additional research is needed also to test the phenocopy and gene-environment covariation models with respect to the association between $H L A-B$ matching and schizophrenia.

A third area of research would focus on hypotheses that hypoxia is the prominent schizophrenia-producing effect of $R H D / H L A-B$ maternal-fetal genotype incompatibility. As examples, if the schizophrenia risk effect of RHD maternalfetal genotype incompatibility is the result of hypoxia, then one would expect to observe an interaction between RHD maternal-fetal genotype incompatibility and hypoxiaregulated/vascular-expression genes. The same hypothesis can be tested with HLA-B maternal-fetal genotype incompatibility. If the schizophrenia risk effect of RHD maternalfetal genotype incompatibility is the result of hypoxia, then one would expect to observe smaller hippocampal volume in individuals with schizophrenia exposed to $R H D$ maternal-fetal genotype incompatibility compared to those not exposed. The same hypothesis can be tested with $H L A-B$ maternal-fetal genotype incompatibility. If the schizophrenia risk effect of $R H D$ maternal-fetal genotype incompatibility is the result of hypoxia, then one would expect $R H D$ maternal-fetal genotype incompatibility to be associated with neurocognitive functions that may be sensitive to the effects of prenatal hypoxia in schizophrenia, for example, verbal learning and memory. The same hypothesis can be tested with $H L A-B$ maternal-fetal genotype incompatibility.

A fourth area of research would focus on hypotheses to further examine offspring sex-dependent differences in the schizophrenia-producing effects of RHD maternal-fetal genotype incompatibility. For this area of research, hypotheses regarding sex-dependent differences in amount of exposure (threshold effect), gestational timing of exposure (timing effect), and type of exposure, that is, hypoxia and hyperbilirubinemia (specific effect) are likely best tested using animal models which can systematically vary conditions of hypoxia and hyperbilirubinemia. Similar investigations can be performed when the prenatal effects of $H L A-B$ maternalfetal genotype incompatibility are better elucidated.

A fifth area of research would focus on identifying other "incompatibility" genes. The attributable risk associated with these maternal-fetal genotype incompatibilities is limited to populations in which the incompatibility occurs with appreciable frequency. As one example, the Rhesus D negative allele is less common in African and Asian populations than European Caucasian populations $[72,120,121]$, hence $R H D$ maternal-fetal genotype incompatibility is less likely to contribute to schizophrenia susceptibility in those populations. However, $R H D$ is not the only blood antigen locus for which a maternal-fetal genotype incompatibility could arise. Other blood antigens exist, including $A B O$ [48], RHCE [122], Kell $[123,124]$, Duffy [125], Kidd [48], and MN [125, 126] and maternal-fetal genotype incompatibilities for these antigens can give rise to a maternal immune response that is similar, although smaller in magnitude, to the RHD incompatibility response. In addition, other genes that could lead to fetal hypoxia, hyperbilirubinemia, or other prenatal conditions associated with schizophrenia, whether through maternalfetal genotype incompatibility, maternal genetic effects alone, or fetal genetic effects alone, should be examined.

\section{Conclusion}

Prenatal environmental factors are quite heterogeneous and difficult to document reliably, making it difficult to test the role of environmental insults in the pathogenesis of schizophrenia. However, there is growing evidence that many prenatal/obstetric complications have a genetic basis, and one stream of research has focused on identifying combinations of maternal-fetal genotypes, that is, maternalfetal genotype incompatibilities, that predispose to prenatal/obstetric complications. Maternal-fetal genotype incompatibility can occur when maternal and fetal genotypes differ, for example, $R H D$ maternal-fetal genotype incompatibility, or when they are too similar, for example, HLA-B maternalfetal genotype incompatibility. Thus far, the $R H D, A B O$, and $H L A-B$ genes have been implicated as risk factors for schizophrenia, with increasing evidence that male and female offspring may be differentially vulnerable to the effects of maternal-fetal genotype combinations involving these genes. A growing number of studies demonstrate that an interaction between prenatal/obstetric complications and putative susceptibility genes increases risk for schizophrenia. Thus, these maternal-fetal genotype incompatibilities are likely to be part of a complex mixture of factors (genetic and environmental), which together act on the brain in ways yet to be identified to result in schizophrenia. The empiric data demonstrating a relationship between these maternalfetal genotype incompatibilities and schizophrenia provide hypotheses for future investigations to further our understanding of their role in increasing risk of schizophrenia.

Until recently, studies to understand the role of maternalfetal genotype incompatibility in schizophrenia (or any complex disorder) have inferred immunologically relevant genotypes solely from birth records and for the single phenomenon of hemolytic disease. As illustrated in this review, maternal-fetal genotype incompatibility at other loci, such as HLA loci, may also increase risk for schizophrenia. However, because these loci do not result in hemolytic disease of the newborn it may be challenging, a priori, to examine their role through information gleaned from birth records. Hence, the development of study designs and statistical methods to study prenatal risk factors based on 
genotype data are essential for further delineating maternalfetal genotype incompatibility as a non-Mendelian mechanism in complex disease. In fact, genetic studies that do not model non-Mendelian patterns of inheritance directly may be one contributing reason that current genome scans have not found striking and highly replicable results in complex disorders that otherwise are so highly familial.

The approach described here integrates the investigation of genes and environment in an innovative manner and provides empirical data that fits within and can be further tested in a genetic-inflammatory-vascular hypothesis of schizophrenia. There are several reasons why it is important to further investigate maternal-fetal genotype incompatibly as a risk factor for schizophrenia: (1) it is a new research approach that allows precise identification of a putative high-risk prenatal environment, even years after the adverse environment has occurred; (2) using a genetic approach, it is possible to simultaneously evaluate alternative explanations of allelic effects that act solely through the genotype of the mother or child; (3) if certain maternal-fetal genotype incompatibilities, for example, $R H D$, do increase risk for schizophrenia, then efforts could be launched to increase prevention of the effects of this class of risk factor; (4) this approach could serve as a model for studying other complex disorders for which maternal-fetal genotype incompatibilities may be involved, for example, diabetes [127, 128] and rheumatoid arthritis [129-131].

\section{References}

[1] M. K. Spearing, “Overview of schizophrenia," NIH Publication 02-3517, National Institutes of Health, Bethesda, Md, USA, 2002.

[2] A. J. Mitchell and D. Malone, "Physical health and schizophrenia," Current Opinion in Psychiatry, vol. 19, no. 4, pp. 432-437, 2006.

[3] B. A. Palmer, V. S. Pankratz, and J. M. Bostwick, "The lifetime risk of suicide in schizophrenia: a reexamination," Archives of General Psychiatry, vol. 62, no. 3, pp. 247-253, 2005.

[4] American Psychiatric Association, Diagnostic and Statistical Manual, American Psychiatric Association, Washington, DC, USA, 4th edition, 1994.

[5] A. Aleman, R. S. Kahn, and J.-P. Selten, "Sex differences in the risk of schizophrenia: evidence from meta-analysis," Archives of General Psychiatry, vol. 60, no. 6, pp. 565-571, 2003.

[6] A. Thorup, B. L. Waltoft, C. B. Pedersen, P. B. Mortensen, and M. Nordentoft, "Young males have a higher risk of developing schizophrenia: a Danish register study," Psychological Medicine, vol. 37, no. 4, pp. 479-484, 2007.

[7] D. J. Castle and R. M. Murray, "The neurodevelopmental basis of sex differences in schizophrenia," Psychological Medicine, vol. 21, no. 3, pp. 565-575, 1991.

[8] K. T. Mueser and S. R. McGurk, "Schizophrenia," The Lancet, vol. 363, no. 9426, pp. 2063-2072, 2004.

[9] J. E. Salem and A. M. Kring, "The role of gender differences in the reduction of etiologic heterogeneity in schizophrenia," Clinical Psychology Review, vol. 18, no. 7, pp. 795-819, 1998.

[10] A. Leung and P. Chue, "Sex differences in schizophrenia, a review of the literature," Acta Psychiatrica Scandinavica, vol. 101, no. 401, pp. 3-38, 2000.

[11] P. F. Sullivan, K. S. Kendler, and M. C. Neale, "Schizophrenia as a complex trait: evidence from a meta-analysis of twin studies," Archives of General Psychiatry, vol. 60, no. 12, pp. 1187-1192, 2003.

[12] P. J. Harrison and D. R. Weinberger, "Schizophrenia genes, gene expression, and neuropathology: on the matter of their convergence," Molecular Psychiatry, vol. 10, no. 1, pp. 40-68, 2005.

[13] D. A. Lewis and J. A. Lieberman, "Catching up on schizophrenia: natural history and neurobiology," Neuron, vol. 28, no. 2, pp. 325-334, 2000.

[14] E. Cantor-Graae, B. Ismail, and T. F. McNeil, "Are neurological abnormalities in schizophrenic patients and their siblings the result of perinatal trauma?" Acta Psychiatrica Scandinavica, vol. 101, no. 2, pp. 142-147, 2000.

[15] J. R. Geddes, H. Verdoux, N. Takei, et al., "Schizophrenia and complications of pregnancy and labor: an individual patient data meta-analysis," Schizophrenia Bulletin, vol. 25, no. 3, pp. 413-423, 1999.

[16] H. E. Hulshoff Pol, H. W. Hoek, E. Susser, et al., "Prenatal exposure to famine and brain morphology in schizophrenia," American Journal of Psychiatry, vol. 157, no. 7, pp. 1170-1172, 2000.

[17] T. D. Cannon, "On the nature and mechanisms of obstetric influences in schizophrenia: a review and synthesis of epidemiologic studies," International Review of Psychiatry, vol. 9, no. 4, pp. 387-397, 1997.

[18] T. D. Cannon, I. M. Rosso, J. M. Hollister, C. E. Bearden, L. E. Sanchez, and T. Hadley, "A prospective cohort study of genetic and perinatal influences in the etiology of schizophrenia," Schizophrenia Bulletin, vol. 26, no. 2, pp. 351-366, 2000.

[19] M. Cannon, P. B. Jones, and R. M. Murray, "Obstetric complications and schizophrenia: historical and meta-analytic review," American Journal of Psychiatry, vol. 159, no. 7, pp. 1080-1092, 2002.

[20] M. Cannon and M. C. Clarke, "Risk for schizophreniabroadening the concepts, pushing back the boundaries," Schizophrenia Research, vol. 79, no. 1, pp. 5-13, 2005.

[21] M. C. Clarke, M. Harley, and M. Cannon, "The role of obstetric events in schizophrenia," Schizophrenia Bulletin, vol. 32, no. 1, pp. 3-8, 2006.

[22] H. Verdoux and A.-L. Sutter, "Perinatal risk factors for schizophrenia: diagnostic specificity and relationships with maternal psychopathology," American Journal of Medical Genetics, vol. 114, no. 8, pp. 898-905, 2002.

[23] J. van Os and J.-P. Selten, "Prenatal exposure to maternal stress and subsequent schizophrenia. The May 1940 invasion of The Netherlands," British Journal of Psychiatry, vol. 172, pp. 324-326, 1998.

[24] P. Wright and R. M. Murray, "Schizophrenia: prenatal influenza and autoimmunity," Annals of Medicine, vol. 25, no. 5, pp. 497-502, 1993.

[25] P. Wright, N. Takei, L. Rifkin, and R. M. Murray, "Maternal influenza, obstetric complications, and schizophrenia," American Journal of Psychiatry, vol. 152, no. 12, pp. 17141720, 1995.

[26] A. S. Brown, M. D. Begg, S. Gravenstein, et al., "Serologic evidence of prenatal influenza in the etiology of schizophrenia," Archives of General Psychiatry, vol. 61, no. 8, pp. 774780, 2004.

[27] M. C. Clarke, A. Tanskanen, M. Huttunen, J. C. Whittaker, and M. Cannon, "Evidence for an interaction between familial liability and prenatal exposure to infection in the causation of schizophrenia," American Journal of Psychiatry, vol. 166, no. 9, pp. 1025-1030, 2009. 
[28] E. S. Susser and S. P. Lin, "Schizophrenia after prenatal exposure to the Dutch hunger winter of 1944-1945," Archives of General Psychiatry, vol. 49, no. 12, pp. 983-988, 1992.

[29] D. St. Clair, M. Xu, P. Wang, et al., "Rates of adult schizophrenia following prenatal exposure to the Chinese famine of 1959-1961," Journal of the American Medical Association, vol. 294, no. 5, pp. 557-562, 2005.

[30] A. S. Brown, E. S. Susser, P. D. Butler, R. R. Andrews, C. A. Kaufmann, and J. M. Gorman, "Neurobiological plausibility of prenatal nutritional deprivation as a risk factor for schizophrenia," Journal of Nervous and Mental Disease, vol. 184, no. 2, pp. 71-85, 1996.

[31] M.-Q. Xu, W.-S. Sun, B.-X. Liu, et al., "Prenatal malnutrition and adult Schizophrenia: further evidence from the 19591961 Chinese famine," Schizophrenia Bulletin, vol. 35, no. 3, pp. 568-576, 2009.

[32] J. R. Geddes and S. M. Lawrie, "Obstetric complications and schizophrenia: a meta-analysis," British Journal of Psychiatry, vol. 167, pp. 786-793, 1995.

[33] T. F. McNeil, E. Cantor-Graae, and B. Ismail, "Obstetric complications and congenital malformation in schizophrenia," Brain Research Reviews, vol. 31, no. 2-3, pp. 166-178, 2000.

[34] J. J. McGrath, F. P. Féron, T. H. J. Burne, A. Mackay-Sim, and D. W. Eyles, "The neurodevelopmental hypothesis of schizophrenia: a review of recent developments," Annals of Medicine, vol. 35, no. 2, pp. 86-93, 2003.

[35] S. Marenco and D. R. Weinberger, "The neurodevelopmental hypothesis of schizophrenia: following a trail of evidence from cradle to grave," Development and Psychopathology, vol. 12, no. 3, pp. 501-527, 2000.

[36] O. D. Howes, C. McDonald, M. Cannon, L. Arseneault, J. Boydell, and R. M. Murray, "Pathways to schizophrenia: the impact of environmental factors," International Journal of Neuropsychopharmacology, vol. 7, supplement 1, pp. S7-S13, 2004.

[37] T. G. M. van Erp, P. A. Saleh, I. M. Rosso, et al., "Contributions of genetic risk and fetal hypoxia to hippocampal volume in patients with schizophrenia or schizoaffective disorder, their unaffected siblings, and healthy unrelated volunteers," American Journal of Psychiatry, vol. 159, no. 9, pp. 1514-1520, 2002.

[38] F. Ebner, R. Tepest, I. Dani, et al., "The hippocampus in families with schizophrenia in relation to obstetric complications," Schizophrenia Research, vol. 104, no. 1-3, pp. 71-78, 2008.

[39] K. Schulze, C. McDonald, S. Frangou, et al., "Hippocampal volume in familial and nonfamilial schizophrenic probands and their unaffected relatives," Biological Psychiatry, vol. 53, no. 7, pp. 562-570, 2003.

[40] N. Stefanis, S. Frangou, J. Yakeley, et al., "Hippocampal volume reduction in schizophrenia: effects of genetic risk and pregnancy and birth complications," Biological Psychiatry, vol. 46, no. 5, pp. 697-702, 1999.

[41] V. A. Mittal, L. M. Ellman, and T. D. Cannon, "Geneenvironment interaction and covariation in schizophrenia: the role of obstetric complications," Schizophrenia Bulletin, vol. 34, no. 6, pp. 1083-1094, 2008.

[42] K. K. Nicodemus, S. Marenco, A. J. Batten, et al., "Serious obstetric complications interact with hypoxiaregulated/vascular-expression genes to influence schizophrenia risk," Molecular Psychiatry, vol. 13, no. 9, pp. 873-877, 2008.
[43] D. R. Hanson and I. I. Gottesman, "Theories of schizophrenia: a genetic-inflammatory-vascular synthesis," BMC Medical Genetics, vol. 6, article 7, 2005.

[44] M. Huleihel, H. Golan, and M. Hallak, "Intrauterine infection/inflammation during pregnancy and offspring brain damages: possible mechanisms involved," Reproductive Biology and Endocrinology, vol. 2, article 17, 2004.

[45] A. Preti, "Obstetric complications, genetics and schizophrenia," European Psychiatry, vol. 20, no. 4, p. 354, 2005.

[46] P. Laing, et al., "Disruption of fetal brain development by maternal antibodies as an etiological factor in schizophrenia," in Neural Development and Schizophrenia: Theory and Research, S. A. Mednick and J. M. Hollister, Eds., pp. 215245, Plenum Press, New York, NY, USA, 1995.

[47] C. G. S. Palmer, J. A. Turunen, J. S. Sinsheimer, et al., "RHD maternal-fetal genotype incompatibility increases schizophrenia susceptibility," American Journal of Human Genetics, vol. 71, no. 6, pp. 1312-1319, 2002.

[48] A. C. Guyton, Textbook of Medical Physiology, W. B. Saunders, Philadelphia, Pa, USA, 1981.

[49] J. Bowman, "The management of hemolytic disease in the fetus and newborn," Seminars in Perinatology, vol. 21, no. 1, pp. 39-44, 1997.

[50] T. W. R. Hansen, "Bilirubin oxidation in brain," Molecular Genetics and Metabolism, vol. 71, no. 1-2, pp. 411-417, 2000.

[51] T. W. R. Hansen, "Bilirubin brain toxicity," Journal of Perinatology, vol. 21, pp. S48-S51, 2001.

[52] Y. Amit and T. Brenner, "Age-dependent sensitivity of cultured rat glial cells to bilirubin toxicity," Experimental Neurology, vol. 121, no. 2, pp. 248-255, 1993.

[53] W. D. Rhine, S. P. Schmitter, A. C. Yu, L. F. Eng, and D. K. Stevenson, "Bilirubin toxicity and differentiation of cultured astrocytes," Journal of Perinatology, vol. 19, no. 3, pp. 206211, 1999.

[54] D. R. Cotter, C. M. Pariante, and I. P. Everall, "Glial cell abnormalities in major psychiatric disorders: the evidence and implications," Brain Research Bulletin, vol. 55, no. 5, pp. 585-595, 2001.

[55] H. W. Moises, T. Zoega, and I. I. Gottesman, "The glial growth factors deficiency and synaptic destabilization hypothesis of schizophrenia," BMC Psychiatry, vol. 2, article $8,2002$.

[56] J. M. Bowman, "RhD hemolytic disease of the newborn," The New England Journal of Medicine, vol. 339, no. 24, pp. 1775177, 1998.

[57] G. F. Chavez, J. Mulinare, and L. D. Edmonds, "Epidemiology of Rh hemolytic disease of the newborn in the United States," Journal of the American Medical Association, vol. 265, no. 24, pp. 3270-3274, 1991.

[58] M. de Silva, M. Contreras, and P. L. Mollison, "Failure of passively administered anti-Rh to prevent secondary Rh responses," Vox Sanguinis, vol. 48, no. 3, pp. 178-180, 1985.

[59] J. G. Thornton, C. Page, G. Foote, G. R. Arthur, L. A. D. Tovey, and J. S. Scott, "Efficacy and long term effects of antenatal prophylaxis with anti-D immunoglobulin," British Medical Journal, vol. 298, no. 6689, pp. 1671-1673, 1989.

[60] A. Sacker, D. J. Done, T. J. Crow, and J. Golding, "Antecedents of schizophrenia and affective illness. Obstetric complications," British Journal of Psychiatry, vol. 166, pp. 734-741, 1995.

[61] J. M. Hollister, P. Laing, and S. A. Mednick, "Rhesus incompatibility as a risk factor for schizophrenia in male adults," Archives of General Psychiatry, vol. 53, no. 1, pp. 1924, 1996. 
[62] M. Byrne, R. Browne, N. Mulryan, et al., "Labour and delivery complications and schizophrenia. Case-control study using contemporaneous labour ward records," British Journal of Psychiatry, vol. 176, no. JUN., pp. 531-536, 2000.

[63] R. E. Kendell, K. McInneny, E. Juszczak, and M. Bain, "Obstetric complications and schizophrenia. Two casecontrol studies based on structured obstetric records," British Journal of Psychiatry, vol. 176, pp. 516-522, 2000.

[64] J. M. Hollister and C. Kohler, "Schizophrenia: a long-term consequence of hemolytic disease of the fetus and newborn?" International Journal of Mental Health, vol. 29, no. 4, pp. 3861,2000

[65] P. Kraft, C. G. S. Palmer, A. J. Woodward, et al., "RHD maternal-fetal genotype incompatibility and schizophrenia: extending the MFG test to include multiple siblings and birth order," European Journal of Human Genetics, vol. 12, no. 3, pp. 192-198, 2004.

[66] B. J. Insel, A. S. Brown, M. A. Bresnahan, C. A. Schaefer, and E. S. Susser, "Maternal-fetal blood incompatibility and the risk of schizophrenia in offspring," Schizophrenia Research, vol. 80, no. 2-3, pp. 331-342, 2005.

[67] C. G. S. Palmer, E. Mallery, J. A. Turunen, et al., "Effect of Rhesus D incompatibility on schizophrenia depends on offspring sex," Schizophrenia Research, vol. 104, no. 1-3, pp. 135-145, 2008.

[68] M. C. O’Donovan, N. J. Craddock, and M. J. Owen, "Genetics of psychosis; insights from views across the genome," Human Genetics, vol. 126, no. 1, pp. 3-12, 2009.

[69] M. Gill, G. Donohoe, and A. Corvin, "What have the genomics ever done for the psychoses?" Psychological Medicine, vol. 40, pp. 529-540, 2010.

[70] M. J. Khoury, T. H. Beaty, and B. H. Cohen, Fundamentals of Genetic Epidemiology, Oxford University Press, New York, NY, USA, 1993.

[71] D. R. Weinberger, M. F. Egan, A. Bertolino, et al., "Prefrontal neurons and the genetics of schizophrenia," Biological Psychiatry, vol. 50, no. 11, pp. 825-844, 2001.

[72] J. Mwangi, "Blood group distribution in an urban population of patient targeted blood donors," East African Medical Journal, vol. 76, no. 11, pp. 615-616, 1999.

[73] R. G. Harvey, D. Tills, A. Warlow, et al., "Genetic affinities of the Balts: a study of blood groups, serum proteins and enzymes of Lithuanians in the United Kingdom," Royal Anthropological Institute of Great Britain and Ireland, vol. 18, no. 3, pp. 535-552, 1983.

[74] H. Perl, J. A. Ozolek, J. F. Watchko, and F. B. Mimouni, "Differences in clinical significance of maternal-infant blood group incompatibility in mothers with blood type $\mathrm{O}, \mathrm{A}$, or B," Journal of Pediatrics, vol. 126, no. 2, pp. 322-323, 1995.

[75] A. J. Rawson and N. M. Abelson, "Studies of blood group antibodies. IV. Physicochemical differences between isoantiA,B and isoanti-A or isoanti-B," Journal of Immunology, vol. 85, pp. 640-647, 1960.

[76] J. A. Ozolek, J. F. Watchko, and F. Mimouni, "Prevalence and lack of clinical significance of blood group incompatibility in mothers with blood type A or B," Journal of Pediatrics, vol. 125, no. 1, pp. 87-91, 1994.

[77] B. Ulm, G. Svolba, M. R. Ulm, G. Bernaschek, and S. Panzer, "Male fetuses are particularly affected by maternal alloimmunization to D antigen," Transfusion, vol. 39, no. 2, pp. 169-173, 1999.

[78] L. Cahill, "Why sex matters for neuroscience," Nature Reviews Neuroscience, vol. 7, no. 6, pp. 477-484, 2006.
[79] C. M. Hultman, P. Sparén, N. Takei, R. M. Murray, and S. Cnattingius, "Prenatal and perinatal risk factors for schizophrenia, affective psychosis, and reactive psychosis of early onset: case-control study," British Medical Journal, vol. 318, no. 7181, pp. 421-426, 1999.

[80] J. M. Goldstein, L. J. Seidman, S. L. Buka, et al., "Impact of genetic vulnerability and hypoxia on overall intelligence by age 7 in offspring at high risk for schizophrenia compared with affective psychoses," Schizophrenia Bulletin, vol. 26, no. 2, pp. 323-334, 2000.

[81] J. S. Sinsheimer, C. G. S. Palmer, and J. A. Woodward, "Detecting genotype combinations that increase risk for disease: the maternal-fetal genotype incompatibility test," Genetic Epidemiology, vol. 24, no. 1, pp. 1-13, 2003.

[82] H.-J. Hsieh, C. G. S. Palmer, and J. S. Sinsheimer, "Allowing for missing data at highly polymorphic genes when testing for maternal, offspring and maternal-fetal genotype incompatibility effects," Human Heredity, vol. 62, no. 3, pp. 165174, 2006.

[83] H.-J. Hsieh, C. G. S. Palmer, S. Harney, et al., "The vMFG test: investigating maternal, offspring and maternalfetal genetic incompatibility effects on disease and viability," Genetic Epidemiology, vol. 30, no. 4, pp. 333-347, 2006.

[84] S. L. Minassian, C. G. S. Palmer, and J. S. Sinsheimer, "An exact maternal-fetal genotype incompatibility (MFG) test," Genetic Epidemiology, vol. 28, no. 1, pp. 83-95, 2005.

[85] S. L. Minassian, C. G.S. Palmer, J. A. Turunen, et al., "Incorporating serotypes into family based association studies using the MFG test," Annals of Human Genetics, vol. 70, no. 4, pp. 541-553, 2006.

[86] A. Sette, S. Buus, and S. Colon, "Structural characteristics of an antigen required for its interaction with Ia and recognition by T cells," Nature, vol. 328, no. 6129, pp. 395-399, 1987.

[87] P. Wright, V. L. Nimgaonkar, P. T. Donaldson, and R. M. Murray, "Schizophrenia and HLA: a review," Schizophrenia Research, vol. 47, no. 1, pp. 1-12, 2001.

[88] C. Ober, "HLA and pregnancy: the paradox of the fetal allograft," American Journal of Human Genetics, vol. 62, no. 1, pp. 1-5, 1998.

[89] H. Beydoun and A. F. Saftlas, "Association of human leucocyte antigen sharing with recurrent spontaneous abortions," Tissue Antigens, vol. 65, no. 2, pp. 123-135, 2005.

[90] C. Ober, T. Hyslop, S. Elias, L. R. Weitkamp, and W. W. Hauck, "Human leukocyte antigen matching and fetal loss: results of a 10 year prospective study," Human Reproduction, vol. 13, no. 1, pp. 33-38, 1998.

[91] A. M. Unander and L. B. Olding, "Habitual abortion: parental sharing of HLA antigens, absence of maternal blocking antibody, and suppression of maternal lymphocytes," American Journal of Reproductive Immunology, vol. 4, no. 4, pp. 171-178, 1983.

[92] C. L. Ober, A. O. Martin, and J. L. Simpson, "Shared HLA antigens and reproductive performance among Hutterites," American Journal of Human Genetics, vol. 35, no. 5, pp. 9941004, 1983.

[93] S. Fujisawa, "HLA antigens-antibodies system and its association with severe toxemia of pregnancy," Nippon Sanka Fujinka Gakkai Zasshi, vol. 37, no. 1, pp. 124-130, 1985.

[94] P. F. Bolis, M. Martinetti Bianchi, and A. La Fianza, "Immunogenetic aspects of preeclampsia," Biological Research in Pregnancy and Perinatology, vol. 8, no. 1, pp. 42-45, 1987. 
[95] K. Schneider, F. Knutson, L. Tamsen, and O. Sjoberg, "HLA antigen sharing in preeclampsia," Gynecologic and Obstetric Investigation, vol. 37, no. 2, pp. 87-90, 1994.

[96] I. de Luca Brunori, L. Battini, M. Simonelli, et al., "Increased HLA-DR homozygosity associated with pre-eclampsia," Human Reproduction, vol. 15, no. 8, pp. 1807-1812, 2000.

[97] D. Larizza, M. Martinetti, J. M. Dugoujon, et al., "Parental GM an HLA genotypes and reduced birth weight in patients with Turner's syndrome," Journal of Pediatric Endocrinology and Metabolism, vol. 15, no. 8, pp. 1183-1190, 2002.

[98] C. Ober, J. L. Simpson, M. Ward, et al., "Prenatal effects of maternal-fetal HLA compatibilty," American Journal of Reproductive Immunology and Microbiology, vol. 15, no. 4, pp. 141-149, 1987.

[99] M. S. Verp, M. Sibul, C. Billstrand, G. Belen, M. Hsu, and C. Ober, "Maternal-fetal histocompatibility in intrauterine growth retarded and normal weight babies," American Journal of Reproductive Immunology, vol. 29, no. 4, pp. 195-198, 1993.

[100] M. F. Reznikoff-Etievant, J. C. Bonneau, D. Alcalay, et al., "HLA antigen-sharing in couples with repeated spontaneous abortions and the birthweight of babies in successful pregnancies," American Journal of Reproductive Immunology, vol. 25, no. 1, pp. 25-27, 1991.

[101] L. D. Cowan, L. Hudson, G. Bobele, I. Chancellor, and J. Baker, "Maternal-fetal HLA sharing and risk of newborn encephalopathy and seizures: a pilot study," Journal of Child Neurology, vol. 9, no. 2, pp. 173-177, 1994.

[102] C. Dalman, P. Allebeck, J. Cullberg, C. Grunewald, and M. Köster, "Obstetric complications and the risk of schizophrenia: a longitudinal study of a National Birth Cohort," Archives of General Psychiatry, vol. 56, no. 3, pp. 234-240, 1999.

[103] L. Rifkin, S. Lewis, P. Jones, B. Toone, and R. Murray, "Low birth weight and schizophrenia," British Journal of Psychiatry, vol. 165, pp. 357-362, 1994.

[104] R. E. Kendell, E. Juszczak, and S. K. Cole, "Obstetric complications and schizophrenia: a case control study based on standardised obstetric records," British Journal of Psychiatry, vol. 168, pp. 556-561, 1996.

[105] S. L. Buka, M. T. Tsuang, and L. P. Lipsitt, "Pregnancy/delivery complications and psychiatric diagnosis: a prospective study," Archives of General Psychiatry, vol. 50, no. 2, pp. 151-156, 1993.

[106] D. Cudihy and R. V. Lee, "The pathophysiology of preeclampsia: current clinical concepts," Journal of Obstetrics and Gynaecology, vol. 29, no. 7, pp. 576-582, 2009.

[107] E. G. Stubbs, E. R. Ritvo, and A. Mason-Brothers, "Autism and shared parental HLA antigens," Journal of the American Academy of Child Psychiatry, vol. 24, no. 2, pp. 182-185, 1985.

[108] C. Wedekind, T. Seebeck, F. Bettens, and A. J. Paepke, "MHCdependent mate preferences in humans," Proceedings of the Royal Society B, vol. 260, no. 1359, pp. 245-249, 1995.

[109] C. Wedekind and S. Furi, "Body odour preferences in men and women: do they aim for specific MHC combinations or simply heterozygosity?" Proceedings of the Royal Society B, vol. 264, no. 1387, pp. 1471-1479, 1997.

[110] R. Thornhill, S. W. Gangestad, R. Miller, G. Scheyd, J. K. McCollough, and M. Franklin, "Major histocompatibility complex genes, symmetry, and body scent attractiveness in men and women," Behavioral Ecology, vol. 14, no. 5, pp. 668678, 2003.

[111] P. S. C. Santos, J. A. Schinemann, J. Gabardo, and M. Da Graça Bicalho, "New evidence that the MHC influences odor perception in humans: a study with 58 Southern Brazilian students," Hormones and Behavior, vol. 47, no. 4, pp. 384388, 2005.

[112] B. M. Pause, K. Krauel, C. Schrader, et al., "The human brain is a detector of chemosensorily transmitted HLA-class I-similarity in same- and opposite-sex relations," Proceedings of the Royal Society B, vol. 273, no. 1585, pp. 471-478, 2006.

[113] W. J. Brewer, S. J. Wood, C. Pantelis, G. E. Berger, D. L. Copolov, and P. D. McGorry, "Olfactory sensitivity through the course of psychosis: relationships to olfactory identification, symptomatology and the schizophrenia odour," Psychiatry Research, vol. 149, no. 1-3, pp. 97-104, 2007.

[114] P. J. Moberg, R. Agrin, R. E. Gur, R. C. Gur, B. I. Turetsky, and R. L. Doty, "Olfactory dysfunction in schizophrenia: a qualitative and quantitative review," Neuropsychopharmacology, vol. 21, no. 3, pp. 325-340, 1999.

[115] B. I. Turetsky, C. G. Kohler, R. E. Gur, and P. J. Moberg, "Olfactory physiological impairment in first-degree relatives of schizophrenia patients," Schizophrenia Research, vol. 102, no. 1-3, pp. 220-229, 2008.

[116] L. C. Kopala, K. P. Good, K. Morrison, A. S. Bassett, M. Alda, and W. G. Honer, "Impaired olfactory identification in relatives of patients with familial schizophrenia," American Journal of Psychiatry, vol. 158, no. 8, pp. 1286-1290, 2001.

[117] C. G. S. Palmer, H.-J. Hsieh, E. F. Reed, et al., "HLAB maternal-fetal genotype matching increases risk of schizophrenia," American Journal of Human Genetics, vol. 79, no. 4, pp. 710-715, 2006.

[118] J. Havlicek and S. C. Roberts, "MHC-correlated mate choice in humans: a review," Psychoneuroendocrinology, vol. 34, no. 4, pp. 497-512, 2009.

[119] L. J. Vatten and R. Skjaerven, "Offspring sex and pregnancy outcome by length of gestation," Early Human Development, vol. 76, no. 1, pp. 47-54, 2004.

[120] J. Y. Kim, S. Y. Kim, C. A. Kim, G. S. Yon, and S. S. Park, "Molecular characterization of D- Korean persons: development of a diagnostic strategy," Transfusion, vol. 45, no. 3, pp. 345-352, 2005.

[121] H. Okuda, M. Kawano, S. Iwamoto, et al., "The RHD gene is highly detectable in RhD-negative Japanese donors," Journal of Clinical Investigation, vol. 100, no. 2, pp. 373-379, 1997.

[122] S. Mitchell and A. James, "Severe hemolytic disease from rhesus anti-C antibodies in a surrogate pregnancy after oocyte donation: a case report," Journal of Reproductive Medicine for the Obstetrician and Gynecologist, vol. 44, no. 4, pp. 388-390, 1999.

[123] A. Babinszki, R. H. Lapinski, and R. L. Berkowitz, "Prognostic factors and management in pregnancies complicated with severe Kell alloimmunization: experiences of the last 13 years," American Journal of Perinatology, vol. 15, no. 12, pp. 695-701, 1998.

[124] S. Lee, D. Russo, and C. M. Redman, "The Kell blood group system: Kell and XK membrane proteins," Seminars in Hematology, vol. 37, no. 2, pp. 113-121, 2000.

[125] O. Geifman-Holtzman, M. Wojtowycz, E. Kosmas, and R. Artal, "Female alloimmunization with antibodies known to cause hemolytic disease," Obstetrics and Gynecology, vol. 89, no. 2, pp. 272-275, 1997.

[126] D. J. Thompson, D. Z. Stults, and S. J. Daniel, "Anti-M antibody in pregnancy," Obstetrical and Gynecological Survey, vol. 44, no. 9, pp. 637-641, 1989.

[127] G. Dahlquist and B. Kallen, "Maternal-child blood group incompatibility and other perinatal events increase the risk for early-onset type 1 (insulin-dependent) diabetes mellitus," Diabetologia, vol. 35, no. 7, pp. 671-675, 1992. 
[128] G. G. Dahlquist, C. Patterson, and G. Soltesz, "Perinatal risk factors for childhood type I diabetes in Europe: the EURODIAB Substudy 2 Study Group," Diabetes Care, vol. 22, no. 10, pp. 1698-1702, 1999.

[129] S. Ten Wolde, F. C. Breedveld, R. R. P. De Vries, et al., "Influence of non-inherited maternal HLA antigens on occurrence of rheumatoid arthritis," The Lancet, vol. 341, no. 8839, pp. 200-202, 1993.

[130] I. E. Van der Horst-Bruinsma, J. M. W. Hazes, G. M. Th. Schreuder, et al., "Influence of non-inherited maternal HLA-DR antigens on susceptibility to rheumatoid arthritis," Annals of the Rheumatic Diseases, vol. 57, no. 11, pp. 672-675, 1998.

[131] S. Harney, J. Newton, A. Milicic, M. A. Brown, and B. P. Wordsworth, "Non-inherited maternal HLA alleles are associated with rheumatoid arthritis," Rheumatology, vol. 42, no. 1, pp. 171-174, 2003. 

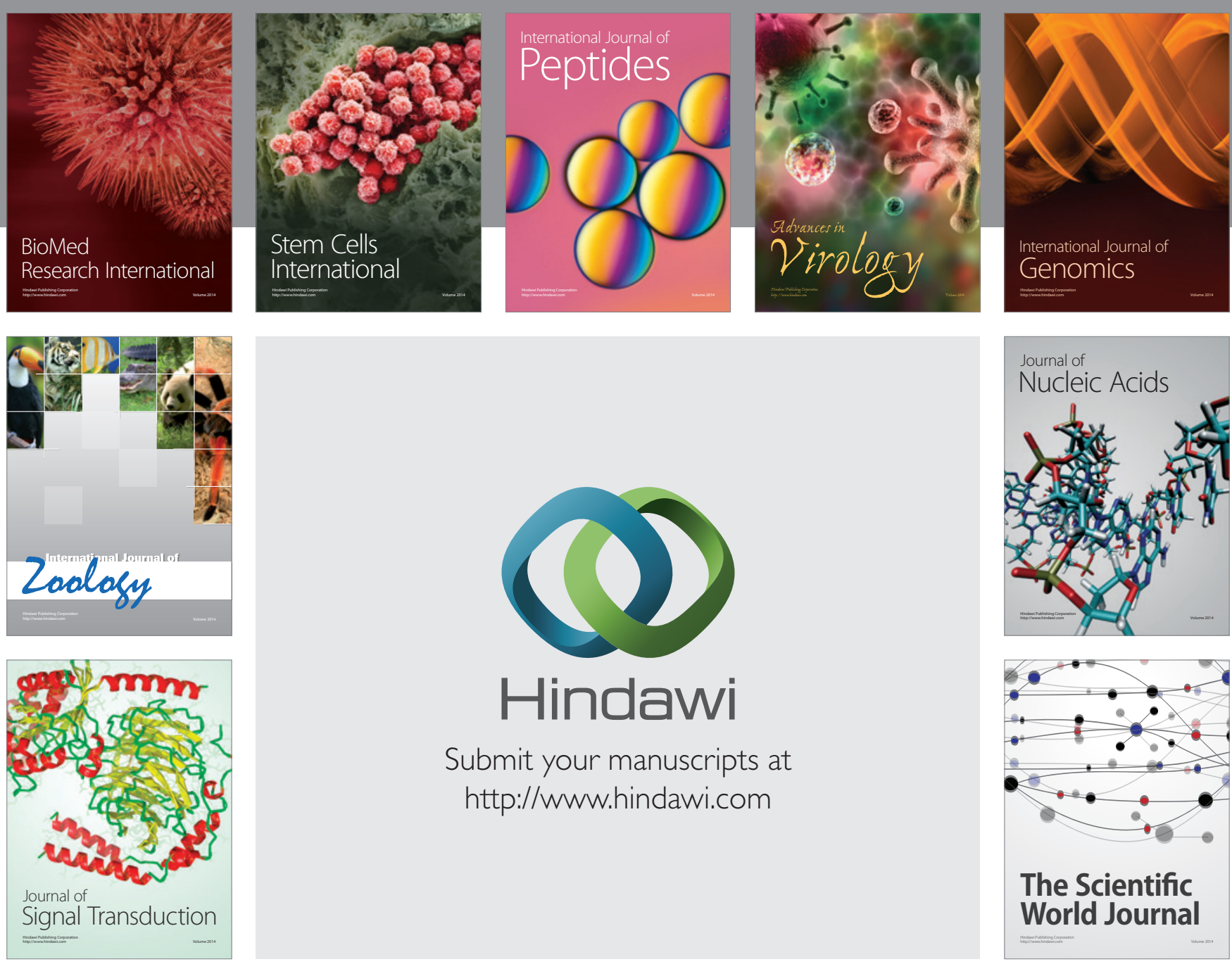

Submit your manuscripts at

http://www.hindawi.com
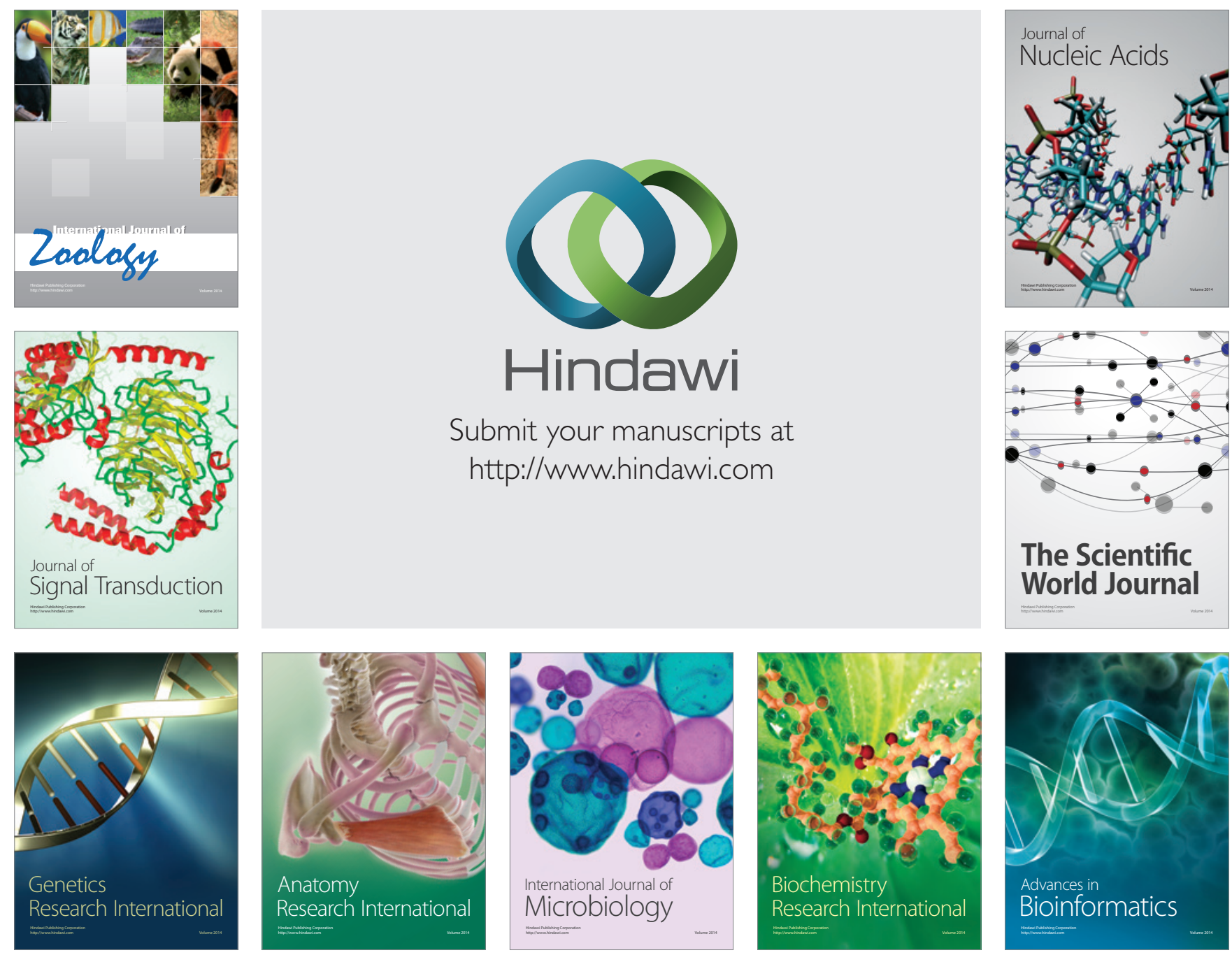

The Scientific World Journal
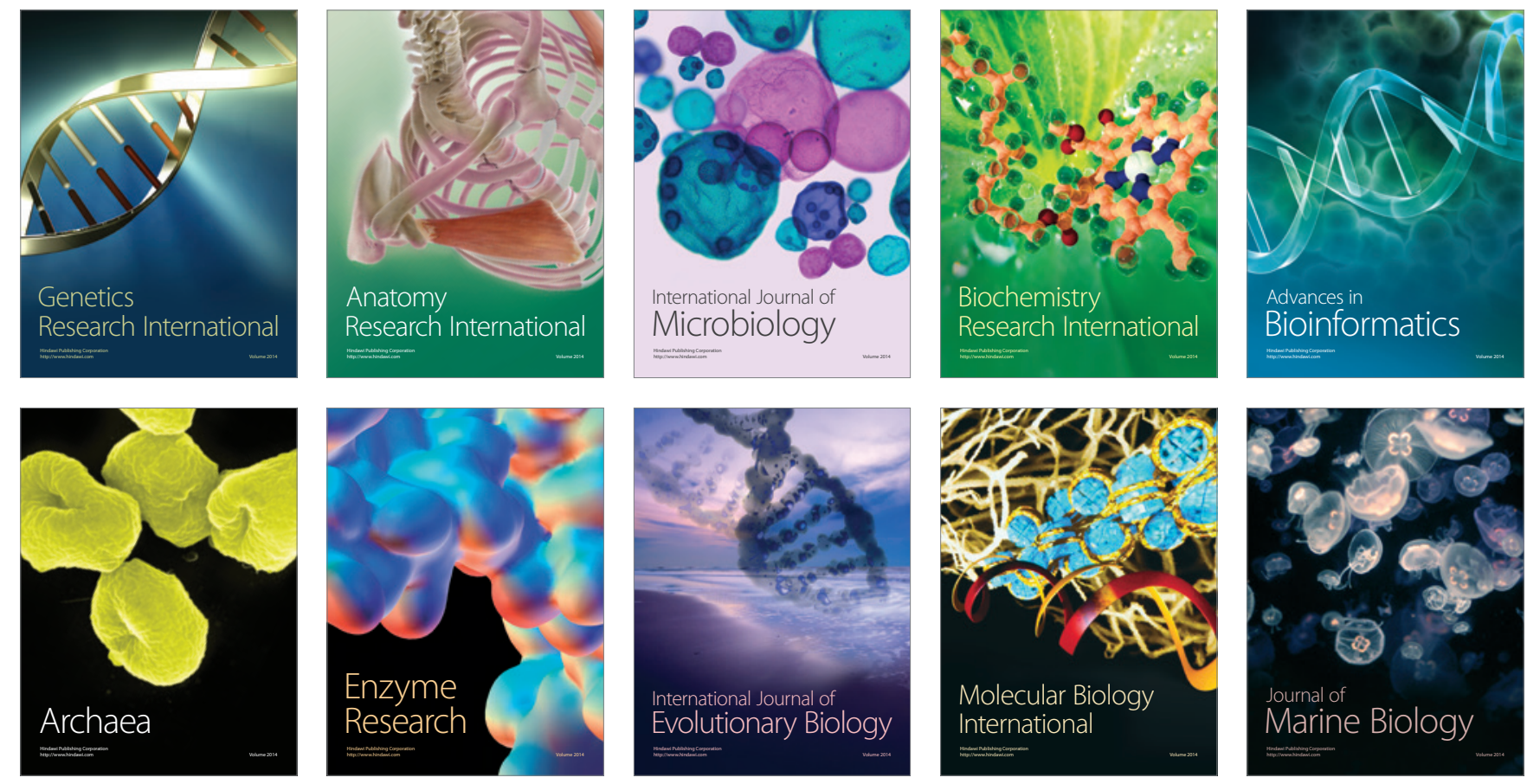